\title{
Thromboelastometry in patients with advanced chronic liver disease stratified by severity of portal hypertension
}

\author{
Pierre Raeven ${ }^{1}$. Joanna Baron-Stefaniak ${ }^{1}$ - Benedikt Simbrunner ${ }^{2,3} \cdot$ Alexander Stadlmann $^{2,3}$. Philipp Schwabl ${ }^{2,3}$. \\ Bernhard Scheiner $^{2,3} \cdot$ Eva Schaden $^{1} \cdot$ Ernst Eigenbauer $^{4} \cdot$ Peter Quehenberger $^{5} \cdot$ Mattias Mandorfer $^{2,3}$. \\ David Marek Baron ${ }^{1} \cdot$ Thomas Reiberger ${ }^{2,3}$ (1)
}

Received: 20 June 2020 / Accepted: 6 September 2020 / Published online: 30 September 2020

(c) The Author(s) 2020

\begin{abstract}
Background Rotational thromboelastometry (ROTEM) has been studied in patients with advanced chronic liver disease (ACLD) without considering the impact of portal hypertension. We evaluated the influence of the hepatic venous pressure gradient (HVPG) on ROTEM results in patients with ACLD.

Methods Cross-sectional study; ACLD patients undergoing HVPG measurement within the prospective Vienna Cirrhosis Study (NCT03267615) underwent concomitant ROTEM testing.

Results Among 159 patients (68\% male; Child-Pugh-A: 53\%, Child-Pugh-B: 34\%, Child-Pugh-C: 13\%), 21 patients (13\%) had a HVPG between 6 and $10 \mathrm{mmHg}, 84$ patients (53\%) between 10 and $19 \mathrm{mmHg}$, and 54 patients $(34 \%) \geq 20 \mathrm{mmHg}$. Child-Pugh-C patients (vs. Child-Pugh-A and vs. Child-Pugh-B patients, respectively) showed longer clot formation time (CFT: median $187 \mathrm{~s}$ vs. $122 \mathrm{~s}$ vs. $122 \mathrm{~s}, p=0.007$ ) and lower maximum clot firmness (MCF: median: $45 \mathrm{~mm}$ vs. $56 \mathrm{~mm}$ vs. $56 \mathrm{~mm}, p=0.002$ ) in extrinsic thromboelastometry (EXTEM), while platelet counts were similar across Child-Pugh stages. In the overall cohort, ROTEM parameters did not differ by severity of portal hypertension. However, among compensated Child-Pugh-A patients, MCF decreased with increasing portal pressure, i.e. in higher HVPG strata (HVPG 9-10 mmHg: median MCF: $59 \mathrm{~mm}$ vs. HVPG 10-19 mmHg: $56 \mathrm{~mm}$ vs HVPG $\geq 20 \mathrm{mmHg}: 54 \mathrm{~mm}, p=0.023$ ). Furthermore, patients with short CFT and high MCF in EXTEM had higher levels of lipopolysaccharide-binding protein, C-reactive protein, and procalcitonin, as well as higher leukocyte counts (all $p<0.05$ ).

Conclusions Portal hypertension seems to impact ROTEM results only in compensated Child-Pugh-A patients. Bacterial translocation and systemic inflammation may trigger a procoagulant state in patients with ACLD.
\end{abstract}

Keywords Cirrhosis $\cdot$ Hepatic venous pressure gradient $\cdot$ Varices $\cdot$ Ascites $\cdot$ Viscoelastic test $\cdot$ Coagulation $\cdot$ Bacterial translocation $\cdot$ Inflammation $\cdot$ Clinical $\cdot$ Hepatic

\section{Introduction}

Electronic supplementary material The online version of this article (https://doi.org/10.1007/s12072-020-10093-3) contains supplementary material, which is available to authorized users.

Thomas Reiberger

thomas.reiberger@meduniwien.ac.at

1 Department of Anaesthesia, Intensive Care Medicine and Pain Medicine, Medical University of Vienna, Vienna, Austria

2 Vienna Hepatic Hemodynamic Lab, Medical University of Vienna, Vienna, Austria
Assessment of hemostasis is challenging in patients with advanced chronic liver disease (ACLD) [1]. Prothrombin time-derived international normalized ratio (PT-INR) and activated partial thromboplastin time (aPTT) are often used

3 Division of Gastroenterology and Hepatology, Department of Medicine III, Medical University of Vienna, Waehringer Guertel 18-20, 1090 Vienna, Austria

4 IT4Science, Medical University of Vienna, Vienna, Austria

5 Department of Laboratory Medicine, Medical University of Vienna, Vienna, Austria 
to evaluate hemostasis and coagulation. Although PT-INR reflects liver synthetic function and is integrated into the Child-Pugh score and Model for end-stage liver disease (MELD), it does not indicate bleeding risk in ACLD patients [2]. This is explained by a concomitant decrease of anticoagulant factors not captured by PT-INR (e.g. antithrombin, protein $\mathrm{C}$, thrombomodulin) and increased levels of von Willebrand Factor and factor VIII [3]. Similarly, aPTT may detect coagulation factor deficiencies or guide heparin therapy but is not associated with disturbed hemostasis in ACLD patients. Of note, PT-INR and aPTT are based on clot formation as an endpoint, which occurs when approximately $5 \%$ of thrombin is generated [4].

Rotational thromboelastometry (ROTEM) is a global viscoelastic coagulation test, validated to guide the treatment of severe bleeding in trauma or major surgical procedures. As a point-of-care test using whole blood, ROTEM represents a more comprehensive test to assess the hemostatic status. Several studies have investigated the use of viscoelastic tests in patients with ACLD from different perspectives [5-10]. Of note, these studies did not assess the influence of hepatic venous pressure gradient (HVPG) or report on the prevalence of clinically significant portal hypertension (CSPH).

CSPH is defined as HVPG $\geq 10 \mathrm{mmHg}$ [11], and is associated with the development of varices and the progression of compensated ACLD (cACLD) to decompensated ACLD $[12,13,14]$. Moreover, $\mathrm{HVPG} \geq 20 \mathrm{mmHg}$ is associated with high risk of bleeding-related mortality $[15,16]$.

In ACLD, CSPH and liver dysfunction facilitate bacterial translocation from the gut to the splanchnic and systemic circulation $[17,18]$. This process can trigger systemic inflammation, reflected by increased circulating levels of lipopolysaccharide-binding protein (LBP), interleukin-6, C-reactive protein (CRP), and procalcitonin, as well as elevated leukocyte counts [17]. Importantly, activation of hemostasis may be a physiological response to bacterial translocation, possibly limiting the dissemination of pathogens [19].

In this study, we examined (i) whether the severity of portal hypertension and hepatic dysfunction affect hemostasis as assessed by ROTEM and (ii) if ROTEM results are associated with parameters related to bacterial translocation.

\section{Patients and methods}

\section{Patient selection}

Data were derived from the prospective Vienna Cirrhosis Study (VICIS). Inclusion criteria were age $\geq 18$ years, diagnosis of ACLD (liver stiffness $>15 \mathrm{kPa}, \mathrm{HVPG}>5 \mathrm{mmHg}$, or histologic F3/F4 fibrosis) and written informed consent. Data sets collected from 336 patients between June 2017 and May 2019 were screened. Exclusion criteria were missing/inconclusive HVPG measurements, missing ROTEM results, presence of acute decompensation, severe alcoholic hepatitis, acute-on-chronic liver failure, hepatocellular carcinoma, liver metastasis, congestive heart failure, previous liver transplantation or transjugular intrahepatic portosystemic shunt. Finally, 159 patients were included in the current study.

Patients under non-selective beta-blockers (NSBB) treatment $(n=30)$ were included in the analysis, as we expected a limited effect of NSBB on ROTEM. NSBB were paused in 26 patients 5 days prior to HVPG measurement. The interruption of NSBB treatment for baseline HVPG measurement is a routine clinical practice at our institution, we are not aware of any bleeding events that occurred in this context. The safety of this approach is also supported by previous studies [20]. Anti-coagulant/anti-platelet medication was paused for HVPG measurements.

\section{Measurement of the hepatic venous pressure gradient (HVPG)}

Hepatic hemodynamic characterization was performed at the Vienna Hepatic Hemodynamic Lab of the Medical University of Vienna in accordance with a standardized operating procedure [21].

\section{Clinical and laboratory parameters}

Demographic characteristics and blood samples were obtained on the day of the HVPG assessment. Child-Pugh score and MELD were calculated based on patients' medical history and laboratory parameters. ROTEM was performed at the Department of Anesthesia, all other laboratory analyses at the Department of Laboratory Medicine (both Medical University of Vienna). Complete blood count and standard biochemical, coagulation, and immunological parameters were analyzed by routine methods.

\section{Rotational thromboelastometry (ROTEM)}

Viscoelastic testing was performed with blood drawn into sodium citrate tubes using the ROTEM delta device (TEM International, Munich, Germany). In the intrinsic thromboelastometry (INTEM), the intrinsic coagulation pathway was activated by ellagic acid, whereas in the extrinsic thromboelastometry (EXTEM), recombinant tissue factor was added to whole blood. In the fibrinogen thromboelastometry (FIBTEM), fibrin polymerization was assessed by adding the platelet inhibitor cytochalasin D to the EXTEM test. We assessed clotting time (CT), defined as the time in seconds until clot initiation; clot formation time (CFT), which indicates the time in seconds from initial clotting until an amplitude of $20 \mathrm{~mm}$ is reached; and maximum clot firmness 
(MCF), which shows the greatest amplitude. For FIBTEM, only MCF was assessed.

\section{Stratification of patients}

Patients were stratified by the severity of liver dysfunction and of portal hypertension into prognostic stages that reflect the risk of mortality. With compensated (cACLD) patients, we discriminated three substages [22]: stage 0 (subclinical portal hypertension [HVPG 6-9] $\mathrm{mmHg}$, no varices), stage 1 (CSPH without varices), and stage 2 (presence of varices). In addition, to explore the correlation of bacterial translocation with ROTEM results at both ends of the distribution, we stratified our cohort into quintiles according to ROTEM values: $0-20 \%$ percentile (Q1), 20-80\% percentile (Q2-4), 80-100\% percentile (Q5).

\section{Statistics}

GraphPad Prism 8 (GraphPad Software, La Jolla, CA, USA) and IBM SPSS Statistics 25 (IBM, Armonk, NY, USA) were used for statistical analyses. Patient characteristics were analyzed using descriptive statistics. Continuous data were expressed as median (interquartile range), while categorical data were expressed as number (proportion) of patients with the specific characteristic. As appropriate, analysis of variance or Kruskal-Wallis test (with Tukey's or Dunn's correction for multiple comparisons), Student's $t$ test and Mann-Whitney $U$ test were applied for group comparisons of continuous variables, while categorical variables were compared using Chi squared test. Correlations were expressed as Spearman's rho. A $p$-value $<0.05$ was defined as statistically significant.

\section{Results}

\section{Patient characteristics (Table 1)}

Patients were clustered based on the Child-Pugh stage and HVPG strata (Fig. 1a). Compensated patients were further grouped according to their prognostic stage (Fig. 1b). In addition, decompensated (i.e. Child-Pugh-B or -C) patients were characterized based on the presence of highrisk portal hypertension: while $51 \%$ of patients had HVPG values $<20 \mathrm{mmHg}$, the remaining $49 \%$ had HVPG values $\geq 20 \mathrm{mmHg}$ (Fig. 1b).

\section{ROTEM results according to Child-Pugh stages (Table 2)}

Initially, selected ROTEM parameters, plasma fibrinogen levels, and platelet counts were evaluated according to
Table 1 Patient characteristics ${ }^{\mathrm{a}}$

\begin{tabular}{lc}
\hline Characteristics & $n(\%)$ \\
\hline Patients & $159(100 \%)$ \\
Age (median years) & 58 \\
Sex (male) & $108(68 \%)$ \\
Etiology & $63(40 \%)$ \\
ALD & $38(24 \%)$ \\
Viral hepatitis & $13(8 \%)$ \\
ALD+ viral hepatitis & $14(9 \%)$ \\
NASH & $5(3 \%)$ \\
Cholestatic & $26(16 \%)$ \\
Other & $159(100 \%)$ \\
Total & \\
HVPG & $21(13 \%)$ \\
6-9 mmHg & $84(53 \%)$ \\
10-19 mmHg & $54(34 \%)$ \\
$\geq 20$ mmHg & $84(53 \%)$ \\
Child-Pugh stage & $54(34 \%)$ \\
A & $21(13 \%)$ \\
B & \\
C & 13 [10-17] \\
MELD Natrium score & 10 [9-15] \\
MELD UNOS score & $1.4[1.2-1.6]$ \\
PT-INR & $36[32-40]$ \\
Albumin (g/l) & $1.1[0.7-2.0]$ \\
Bilirubin (mg/dl) & $0.7[0.6-1.0]$ \\
Creatinine (mg/dl) & \\
\hline a Continuous data are expressed as medians with interquartile range \\
in square brackets. Categorical data are expressed as the number of \\
patients with the specific characteristic (with the percentage in brack- \\
ets). ALD, alcoholic liver disease; NASH, non-alcoholic steatohepa- \\
titis; HVPG, hepatic venous pressure gradient; MELD, Model for \\
end-stage liver disease; UNOS, United Network for Organ Sharing; \\
PT-INR, prothrombin time-derived international normalized ratio \\
\end{tabular}

the Child-Pugh stage (Fig. 1a). In EXTEM, patients with Child-Pugh-C had prolonged CFT (+14\% vs. Child-Pugh$\mathrm{A}, p=0.026 ;+15 \%$ vs. Child-Pugh-B, $p=0.005)$ and reduced $\mathrm{MCF}(-15 \%$ vs. Child-Pugh-A, $p=0.004 ;-16 \%$ vs. Child-Pugh-B, $p=0.002)$. Child-Pugh-C patients had prolonged CT $(+19 \%$ vs. Child-Pugh-A, $p=0.011)$ and CFT $(+49 \%$ vs. Child-Pugh-B, $p=0.009)$ in INTEM and decreased MCF in INTEM (- $15 \%$ vs. Child-PughA, $p=0.001 ;-17 \%$ vs. Child-Pugh-B, $p<0.001)$ and FIBTEM ( $-33 \%$ vs. Child-Pugh-A; $-40 \%$ vs. Child-Pugh-B, both $p<0.001)$. Simultaneously, fibrinogen levels decreased by $35 \%$ and $32 \%$ (both $p<0.001$ ) in Child-Pugh-C vs. Child-Pugh-A and Child-Pugh$B$ patients, respectively. Taken together, severe ACLD (Child-Pugh stage C, as compared to stages A or B) was associated with slower clot initiation and formation, attenuated clot firmness, and lower fibrinogen levels, but not with reduced platelet count. 


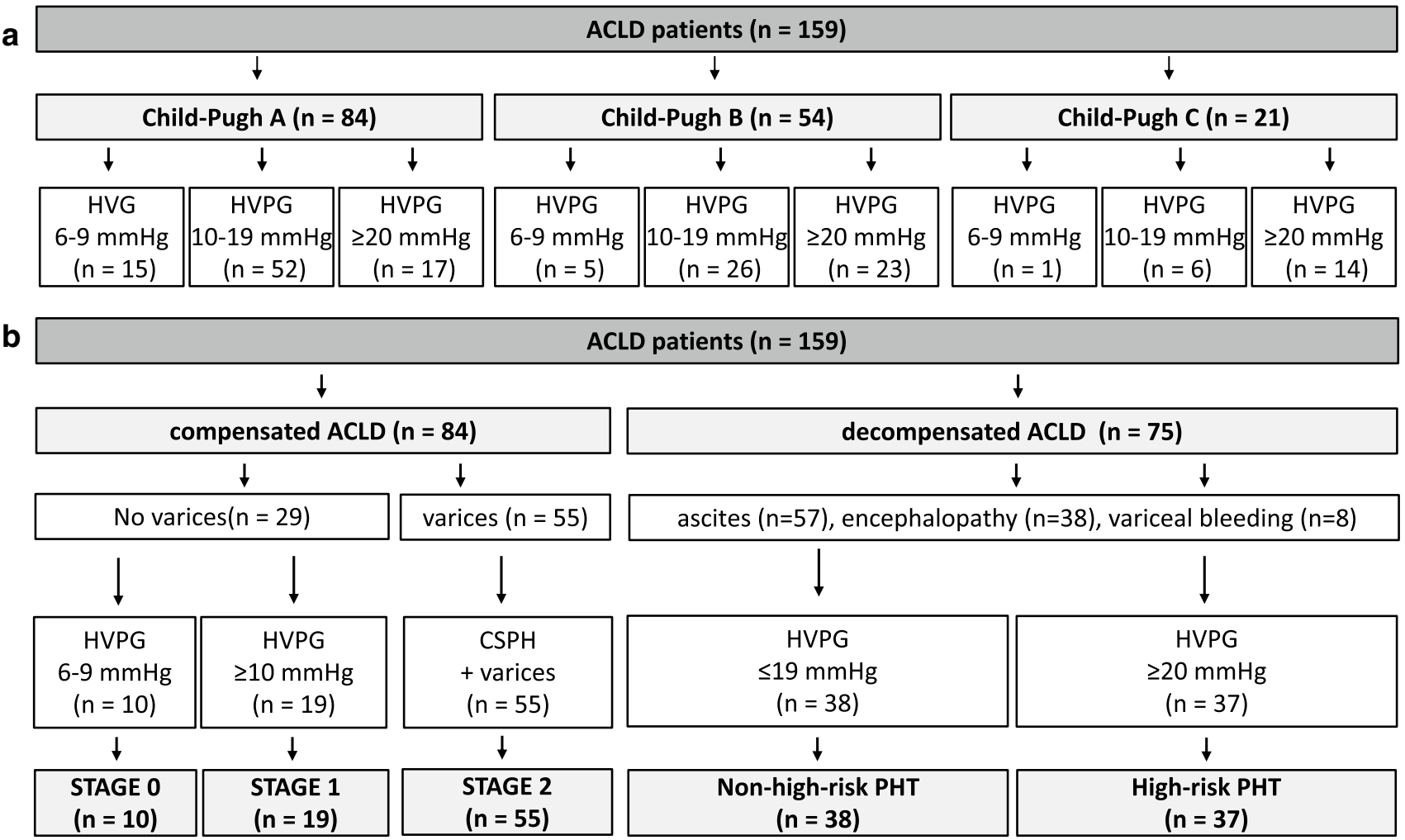

Fig. 1 Patient flow chart. Patients were stratified based on ChildPugh stage. a Within Child-Pugh subcohorts, patients were further grouped based on predefined HVPG cut-offs. b Patients with ChildPugh-A were subdivided into stages 0,1 , and 2 . Patients with ChildPugh-B and $-\mathrm{C}$ were stratified based on the presence or absence of high-risk portal hypertension, as defined by a HVPG $\geq 20 \mathrm{mmHg}$. Some patients had more than one decompensating event. ACLD, advanced chronic liver disease; HVPG: hepatic-venous pressure gradient; PHT, portal hypertension
Table 2 Results of selected ROTEM tests, platelet counts and fibrinogen levels in patients stratified by Child-Pugh status ${ }^{\mathrm{a}}$

\begin{tabular}{lcccr}
\hline & Child-Pugh A & Child-Pugh B & Child-Pugh C & $p$-value \\
\hline EXTEM CT (s) & $74[66-89]$ & $75[66-82]$ & $74[70-93]$ & 0.463 \\
EXTEM CFT (s) & $122[99-200]^{*}$ & $122[82-188]^{* *}$ & $187[133-255]$ & 0.007 \\
EXTEM MCF (mm) & $56[49-59]^{* *}$ & $56[49-62]^{* *}$ & $45[41-52]$ & 0.002 \\
INTEM CT (s) & $192[170-217]^{*}$ & $201[169-224]$ & $227[191-253]$ & 0.014 \\
INTEM CFT (s) & $105[82-173]$ & $105[69-152]^{*}$ & $163[107-214]$ & 0.012 \\
INTEM MCF $(\mathrm{mm})$ & $55[49-59]^{* *}$ & $55[46-61]^{* * *}$ & $44[40-51]$ & $<0.001$ \\
FIBTEM MCF $(\mathrm{mm})$ & $14[11-18]^{* * *}$ & $15[12-20]^{* * *}$ & $10[7-13]$ & $<0.001$ \\
Fibrinogen $(\mathrm{mg} / \mathrm{dl})$ & $288[238-343]^{* * *}$ & $274[219-365]^{* * *}$ & $186[142-235]$ & $<0.001$ \\
Platelets $(\mathrm{G} / \mu \mathrm{l})$ & $99[65-134]$ & $106[71-156]$ & $91[56-102]$ & 0.122 \\
\hline
\end{tabular}

${ }^{\mathrm{a}}$ Data are expressed as medians with interquartile range in square brackets. $n=84$ (Child-Pugh $\mathrm{A}$ ), $n=54$ (Child-Pugh B, except $n=53$ for platelet count), $n=21$ (Child-Pugh C). Data were analyzed using analysis of variance (ANOVA) with Tukey's test for post hoc comparisons (INTEM MCF and fibrinogen) or Kruskal-Wallis test with Dunn's test for post hoc comparisons (remaining parameters). ROTEM, rotational thromboelastometry; EXTEM, extrinsic thromboelastometry; INTEM, intrinsic thromboelastometry; CT, clotting time; CFT, clot formation time; MCF, maximal clot firmness; FIBTEM, fibrinogen thromboelastometry. $* p<0.05$ vs. Child-Pugh C, $* * p<0.01$ vs. Child-Pugh C, $* * * p<0.001$ vs. Child-Pugh C

\section{ROTEM results according to HVPG strata in compensated and decompensated ACLD}

We then analyzed selected ROTEM parameters, plasma fibrinogen levels, and platelet counts according to HVPG strata (Fig. 1a). As these parameters were similar across different HVPG strata in the overall cohort (Table 3), we specifically examined whether HVPG impact on ROTEM 
Table 3 Results of selected ROTEM tests, platelet counts and fibrinogen levels in patients stratified by severity of portal hypertension $^{\mathrm{a}}$

\begin{tabular}{lcccc}
\hline & \multicolumn{2}{l}{ HVPG } & \\
\cline { 2 - 4 } & $6-9 \mathrm{mmHg}$ & $10-19 \mathrm{mmHg}$ & $\geq 20 \mathrm{mmHg}$ & $p$-value \\
\hline EXTEM CT (s) & $77[69-87]$ & $74[66-85]$ & $75[65-88]$ & 0.656 \\
EXTEM CFT (s) & $126[88-174]$ & $128[100-208]$ & $132[90-219]$ & 0.804 \\
EXTEM MCF (mm) & $57[50-65]$ & $55[46-59]$ & $53[44-60]$ & 0.347 \\
INTEM CT (s) & $200[167-233]$ & $198[169-222]$ & $203[180-227]$ & 0.561 \\
INTEM CFT (s) & $103[75-144]$ & $110[82-175]$ & $114[75-201]$ & 0.653 \\
INTEM MCF (mm) & $55[52-64]$ & $55[45-59]$ & $52[44-59]$ & 0.271 \\
FIBTEM MCF (mm) & $13[11-21]$ & $14[10-16]$ & $14[10-18]$ & 0.921 \\
Fibrinogen $(\mathrm{mg} / \mathrm{dl})$ & $313[201-373]$ & $261[216-324]$ & $273[209-341]$ & 0.323 \\
Platelets $(\mathrm{G} / \mu \mathrm{l})$ & $141[74-164]$ & $99[67-132]$ & $96[55-126]$ & 0.173 \\
\hline
\end{tabular}

${ }^{a}$ Data are expressed as medians with interquartile range in square brackets. $n=21$ (HVPG 6-9 $\mathrm{mmHg}$ ), $n=84$ (HVPG $10-19 \mathrm{mmHg}$, except $n=83$ for platelet count), $n=54$ (HVPG $\geq 20 \mathrm{mmHg}$ ). Data were analyzed using analysis of variance (ANOVA) with Tukey's test for post hoc comparisons (EXTEM MCF, INTEM MCF and fibrinogen) or Kruskal-Wallis test with Dunn's test for post hoc comparisons (remaining parameters). ROTEM, rotational thromboelastometry; HVPG, hepatic venous pressure gradient; EXTEM, extrinsic thromboelastometry; INTEM, intrinsic thromboelastometry; CT, clotting time; CFT, clot formation time; MCF, maximal clot firmness; FIBTEM, fibrinogen thromboelastometry results in cACLD patients (Table 4). Within cACLD patients, MCF in EXTEM and INTEM and platelet counts progressively decreased with the severity of portal hypertension (Table 4).

In contrast, within decompensated (Child-Pugh-B/C patients, Table-S2) ACLD patients, ROTEM, fibrinogen levels, and platelet counts were similar across HVPG strata. Similarly, there was no difference in ROTEM results, fibrinogen levels or platelet counts in patients on NSBB (Table-S3) or between subcohorts stratified by severity of portal hypertension (Table-S4).

\section{ROTEM results in patients with various prognostic stages of portal hypertension}

We then analyzed ROTEM results in compensated ACLD patients across substages based on the presence of varices and severity of portal hypertension (Fig. 1b). Although there was a trend towards prolonged CFT in EXTEM and INTEM and impaired MCF in EXTEM, INTEM, and FIBTEM in more advanced compensated ACLD, only INTEM CT was significantly prolonged by $17 \%(p=0.045)$ in stage 2 compared to stage 0 patients (Table-S5). This increase in INTEM CT was associated with decreased platelet counts in stage 2 $(-40 \%, p<0.001)$ and stage $1(-17 \%, p=0.033)$ compared
Table 4 Results of selected ROTEM tests, platelet counts and fibrinogen levels in compensated (Child-Pugh A) patients stratified by severity of portal hypertension $^{\mathrm{a}}$

\begin{tabular}{lcccc}
\hline & \multicolumn{2}{l}{ HVPG } & \\
\cline { 2 - 4 } & $6-9 \mathrm{mmHg}$ & $10-19 \mathrm{mmHg}$ & $\geq 20 \mathrm{mmHg}$ & $p$ value \\
\hline EXTEM CT (s) & $71[66-90]$ & $74[66-87]$ & $76[65-93]$ & 0.895 \\
EXTEM CFT (s) & $109[80-152]$ & $121[102-216]$ & $127[99-232]$ & 0.212 \\
EXTEM MCF (mm) & $59[54-68]$ & $56[48-59]^{*}$ & $54[45-58]^{*}$ & 0.023 \\
INTEM CT (s) & $181[152-215]$ & $192[168-214]$ & $198[177-238]$ & 0.280 \\
INTEM CFT (s) & $101[62-118]$ & $105[83-175]$ & $118[91-220]$ & 0.109 \\
INTEM MCF (mm) & $57[53-67]$ & $55[48-59]^{*}$ & $52[44-55] *$ & 0.009 \\
FIBTEM MCF (mm) & $17[12-23]$ & $13[11-16]$ & $14[12-18]$ & 0.066 \\
Fibrinogen $(\mathrm{mg} / \mathrm{dl})$ & $338[262-388]$ & $281[229-330]$ & $289[235-335]$ & 0.057 \\
Platelets $(\mathrm{G} / \mu \mathrm{l})$ & $143[88-169]$ & $99[65-132]^{*}$ & $84[40-117] *$ & 0.002 \\
\hline
\end{tabular}

${ }^{a}$ Data are expressed as medians with interquartile range in square brackets. $n=15$ (HVPG $6-9 \mathrm{mmHg}$ ), $n=52$ (HVPG $10-19 \mathrm{mmHg}$ ), $n=17$ (HVPG $\geq 20 \mathrm{mmHg}$ ). Data were analyzed using analysis of variance (ANOVA) with Tukey's test for post hoc comparisons (EXTEM MCF, INTEM CT, INTEM MCF, FIBTEM MCF) or Kruskal-Wallis test with Dunn's test for post hoc comparisons (remaining parameters). ROTEM, rotational thromboelastometry; HVPG, hepatic venous pressure gradient; EXTEM, extrinsic thromboelastometry; INTEM, intrinsic thromboelastometry; CT, clotting time; CFT, clot formation time; MCF, maximal clot firmness; FIBTEM, fibrinogen thromboelastometry. ${ }^{*} p<0.05$ vs. HVPG 6-9 mmHg 
to stage 0 cirrhotic patients (Table-S5). Of note, aPTT (stage $0: 35 \mathrm{~s}$; stage $1: 38 \mathrm{~s}$; stage $2: 39 \mathrm{~s} ; p=0.099$ ) was not different between early compensated stages of ACLD.

\section{ROTEM results in decompensated ACLD with or without high-risk portal hypertension}

ROTEM values were compared among dACLD patients stratified by the presence or absence of high-risk portal hypertension (i.e. HVPG $\geq 20 \mathrm{mmHg}$, Fig. 1b). Within the dACLD population, all ROTEM analyses, as well as fibrinogen levels and platelet counts, were not significantly different regardless of the presence of high-risk portal hypertension (Table-S6). This may indicate that within dACLD patients, the degree of liver dysfunction affects ROTEM results to a greater extent than the severity of portal hypertension.

\section{Correlations of ROTEM results with clinical scores and laboratory parameters}

Table-S7 shows a crude correlation matrix of analyzed parameters and ROTEM parameters. Importantly, ROTEM parameters did not correlate with Child-Pugh score and MELD, nor with HVPG or liver stiffness. Fibrinogen levels and platelet counts showed the strongest correlation with MCF and CFT, but not with CT. Concentrations of LBP and leukocyte counts showed a weak to moderate correlation with CFT and MCF in EXTEM, INTEM, and FIBTEM. Levels of CRP were correlated with CFT in EXTEM and INTEM as well as with MCF in FIBTEM.

\section{Analysis of the potential impact of bacterial translocation and systemic inflammation on ROTEM results}

Based on the results observed in the correlation matrix, we evaluated whether patients with ROTEM values in the lowest (Q1) and highest (Q5) quintiles differ in surrogate parameters of bacterial translocation and systemic inflammation (Fig. 2a-l and Fig.S1). Higher levels of LBP (Fig. 2a-c) and higher leukocyte counts (Fig. 2d-f) were associated with shorter CFT and stronger MCF in EXTEM $(p<0.05)$. The impact of systemic inflammation on ROTEM results was underlined by a similar pattern for CRP and procalcitonin (Fig. 2g-1) as for leukocyte count.

We further analyzed these parameters of bacterial translocation and systemic inflammation in the whole cohort and in Child-Pugh B/C patients with or without high-risk portal hypertension (Tables-S8, S9). In the overall cohort, CRP was significantly higher in the patients with highrisk (HVPG $\geq 20 \mathrm{mmHg}$ ) versus non-high-risk (HVPG 6-19 $\mathrm{mmHg}$ ) portal hypertension, but all other parameters were not significantly different.
Of note, 20 patients were treated with rifaximin for encephalopathy, two received antibiotic treatment for secondary prophylaxis after spontaneous bacterial peritonitis (ciprofloxacin and norfloxacin), one received amoxicillin/ clavulanic acid for urinary tract infection and one received trimethoprim/sulfamethoxazole for pneumocystis jirovecii pneumonia prophylaxis.

Taken together, these data indicate that a systemic proinflammatory state and bacterial translocation-often present in patients with cirrhosis-potentially affect clot formation and clot firmness as assessed by ROTEM.

\section{Discussion}

To our knowledge, this is the first study investigating the influence of HVPG on ROTEM parameters in a thoroughly characterized ACLD cohort. First, observed that severe liver dysfunction and low fibrinogen levels are associated with prolonged clot formation and reduced clot firmness. Importantly, by the use of HVPG measurements, we could demonstrate that portal hypertension impairs clot firmness only in compensated patients. In addition, our ROTEM results suggest that pathological bacterial translocation and systemic inflammation are associated with shorter CFT and enhanced MCF and may predispose to a procoagulant state in patients with ACLD.

In our cohort, $87 \%$ of patients had CSPH (HVPG $\geq 10 \mathrm{mmHg}$ ), and a considerable number of patients had decompensated disease ( $47 \%$ with Child-Pugh B/C), indicating more advanced disease as compared to other ACLD patient cohorts [13]. Moreover, a thorough characterisation of HVPG distinguishes our data from previous studies assessing ROTEM [5, 7-10] or thromboelastography (TEG) [6], which did not report HVPG and included mostly compensated patients.

Interestingly, ROTEM results were not associated with the degree of portal hypertension in the entire cohort. In contrast, increased portal pressure was associated with decreased MCF in compensated Child-Pugh-A patients. This finding may be causally related to the coinciding decrease in platelet counts due to hypersplenism secondary to portal hypertension. However, low platelet counts are not associated with increased bleeding risk in cACLD patients, even in those on anti-coagulant medication [23]. Interestingly, a comparable degree of thrombocytopenia was observed across three Child-Pugh stages. This might be explained by a referral bias since among Child-Pugh A patients, those with thrombocytopenia are considerably more likely to be referred to HVPG measurement. Of note, about half of the Child-Pugh-A patients with HVPG $\geq 20 \mathrm{mmHg}$ were previously decompensated. Although HVPG values $\geq 16-20 \mathrm{mmHg}$ are predictive of the development of 
EXTEM CT
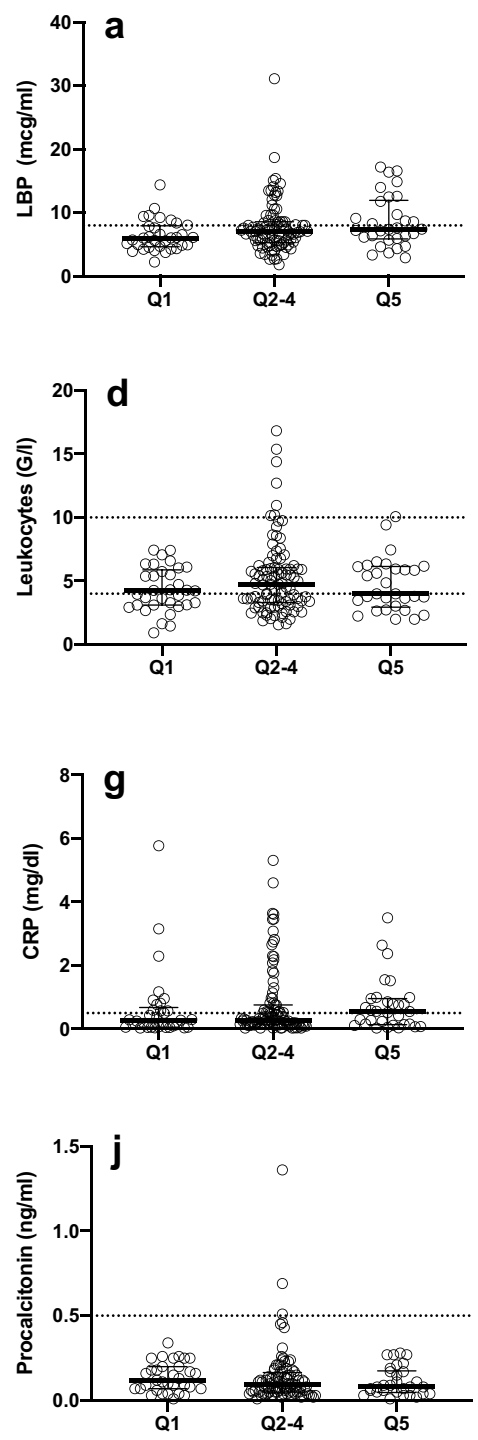

EXTEM CFT
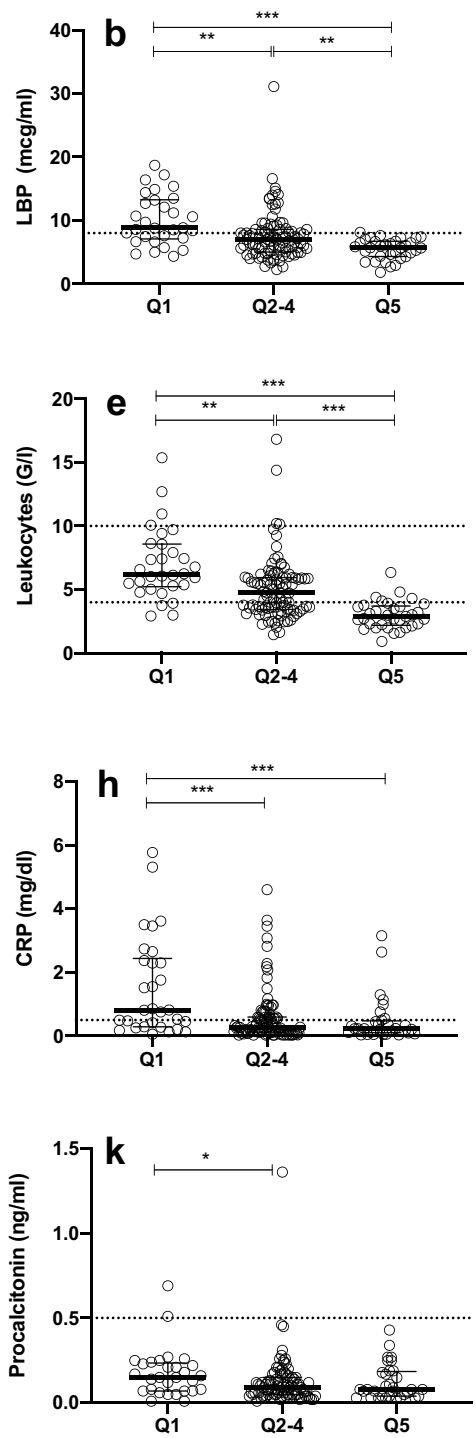

EXTEM MCF
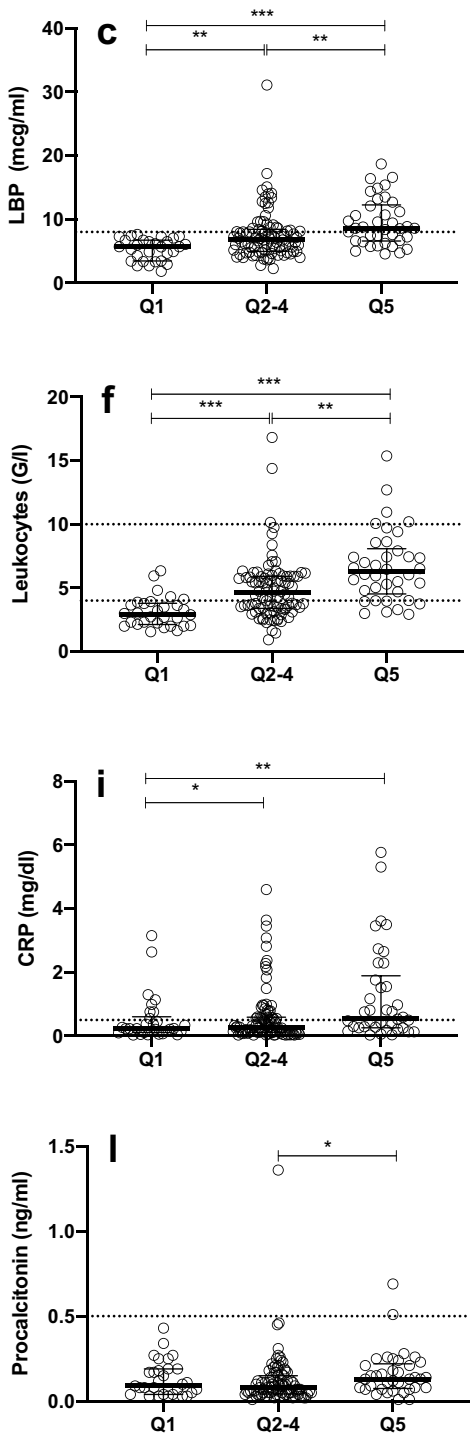

Fig. 2 Impact of bacterial translocation and systemic inflammation on extrinsic thromboelastometry results. Lipopolysaccharide-binding protein in a EXTEM CT, b EXTEM CFT and $\mathbf{c}$ EXTEM MCF strata. Leukocytes in d EXTEM CT, e EXTEM CFT and $\mathbf{f}$ EXTEM MCF strata. C-reactive protein levels in $\mathbf{g}$ EXTEM CT, $\mathbf{h}$ EXTEM CFT and $\mathbf{i}$ EXTEM MCF strata. Procalcitonin levels in $\mathbf{j}$ EXTEM CT, $\mathbf{k}$ EXTEM CFT and (L) EXTEM MCF strata. *p $<0.05$; $* * p<0.01$;

hepatic decompensation in compensated patients [21, 24], HVPG values may exceed $\geq 20 \mathrm{mmHg}$ in compensated patients. For instance, Jindal et al. reported that $19 \%$ of compensated patients had HVPG values $\geq 20 \mathrm{mmHg}$ [24]. To further elucidate the potential impact of portal hypertension on coagulation in compensated patients, we also integrated the presence of varices into our analysis. Therefore, we stratified cACLD patients into substage 0 without CSPH,
$* * * p<0.001$ (analysis of variance/Tukey's or Kruskal-Wallis/ Dunn's). Dotted lines indicate (lower and) upper reference value according to manufacturer/laboratory. EXTEM, extrinsic thromboelastometry; CT, clotting time; CFT, clot formation time; $\mathrm{MCF}$, maximum clot firmness. LPB, lipopolysaccharide-binding protein; CRP, C-reactive protein; Q1, 0-20 percentile; Q2-4, 21-80 percentile; Q5, 81-100 percentile

substage 1 with CSPH but without varices, and substage 2 with CSPH and with varices [22]. Again, a trend towards impaired clot formation was observed from substage 0 to substage 2 of portal hypertension, and lower platelets in substages $1 / 2$ compared to substage 0 . Interestingly, cACLD patients in substage 2 showed a significantly prolonged clotting time in INTEM. There are various potential reasons for this observation: First, this could be an effect of endogenous 
heparinoids. Second, a prolonged INTEM CT in substage 2 might have been influenced by decreased clotting factors. Previous studies indicate that blood tests of synthetic function are linked to the severity of portal hypertension and models including INR have been shown to indicate the presence of CSPH in cACLD patients [25]. However, all of these "easily-available" markers are in many ways inaccurate and the diagnostic and discriminatory value for CSPH (vs. no CSPH) is only moderate. Other-more robust non-invasive markers-are currently being investigated but are not yet applied clinically. While the severity of the liver disease is usually reflected by both HVPG (i.e., extent of portal hypertension) and hepatic impairment, these parameters do not always match in their severity. Furthermore, there is no conclusive evidence that severity of portal hypertension per se impacts on hepatic synthetic function. However, portal hypertension drives bacterial translocation into the systemic circulation, which likely impacts hepatic function by triggering a pro-inflammatory response [18]. Accordingly, the reduced hepatic synthetic function could have contributed to the tendency towards impaired INTEM in stage $1 / 2$ patients. Third, this observation might be the result of a type 1 error.

We then assessed ROTEM results within patients with the most advanced liver disease. Along with Child-Pugh-C and active bleeding at endoscopy, an HVPG of $\geq 20 \mathrm{mmHg}$ is associated with failure to control variceal bleeding and bleeding-related mortality, thus defining high-risk portal hypertension [15]. In contrast to observations in compensated patients, ROTEM results did not differ among decompensated patients with various degrees of portal hypertension (i.e. between HVPG $<20 \mathrm{mmHg}$ vs. $\geq 20 \mathrm{mmHg}$ ). Interestingly, platelet count and fibrinogen levels were not affected by HVPG status. This observation might be explained by a more pronounced impact of biochemical (i.e. impaired synthesis of determinants of hemostasis) rather than mechanical (i.e. portal hypertension) factors on ROTEM results. Decreased thrombopoietin production in dACLD patients may have ameliorated the impact of portal hypertension on platelet counts. However, in our study, their thrombopoietin levels were not significantly different between HVPG subgroups (data not shown).

In clinical practice, ROTEM may not be useful as a predictive tool for (spontaneous) or iatrogenic bleeding, but more as an asset to guide the use of blood products in case of ongoing bleeding. Nevertheless, a hypocoagulable state detected by ROTEM/TEG has been shown in patients who experienced early variceal rebleeding [26] and bleeding during invasive procedures [27].

Since there were few patients across all Child-Pugh stages and all HVPG strata who showed unexpectedly low or high results on ROTEM, we also assessed potential mechanistic factors for this phenomenon. Interestingly, we found that parameters of bacterial translocation (LBP) and systemic inflammation (i.e. leukocyte counts, CRP and PCT) inversely correlated with CFT and positively correlated with MCF. This potential impact of bacterial translocation on ROTEM is especially relevant, since bacterial translocation in ACLD patients is driven by portal hypertension [17] and by liver dysfunction. However, parameters of bacterial translocation and systemic inflammation-except for CRP levels-were similar in patients with low-risk versus highrisk portal hypertension.

We have recently observed a positive correlation between von Willebrand Factor antigen levels as an indicator of endothelial dysfunction and markers of systemic inflammation, independent of HVPG [28]. Elevated thrombin generation capacity and an unbalanced ratio between von Willebrand factor and its cleaving protein (ADAMTS13) have recently been described in cirrhosis [29]. Moreover, increases in CRP are paralleled by increases in factor VIII in patients with ACLD/portal hypertension without active bacterial infection [3]. Overall, this would suggest that bacterial translocation and a proinflammatory state result in a shorter clot formation time and stronger clot firmness, i.e. (inappropriate) promotion of clot formation and stability [30]. Even if this pathophysiologic explanation seems applicable to patients with ACLD, further studies investigating the mechanistic link between bacterial translocation, systemic inflammation and (ROTEM-based assessment of) hemostasis are warranted.

Our study has some limitations: First, we focused on the influence of portal hypertension on ROTEM results in clinically stable outpatients. Therefore, clinical outcome parameters such as bleeding, the subsequent occurrence of (further) hepatic decompensation, thrombo-embolic events, transplantation, or mortality were not assessed. Second, the study might be underpowered for some analyses and may suffer from type 2 error despite including 159 patients. Third, we did not rule out dysfibrinogenemia in our cohort, which could be a reason for delayed clot initiation and attenuated firmness despite sufficiently high fibrinogen levels.

In conclusion, our systemic ROTEM measurements demonstrated prolonged clot formation time and impaired clot firmness in patients with Child-Pugh stage B/C liver dysfunction. Interestingly, portal hypertension impacted ROTEM results only in compensated ACLD patients, with impaired clot firmness when CSPH is present. Mechanistically, bacterial translocation, and systemic inflammation may potentially trigger a procoagulant state in patients with ACLD.

Acknowledgements We thank all co-workers involved in sample preparation and analysis. Specifically, the nurses of the Vienna Hepatic Hemodynamic Lab and the technicians at the Department of Anaesthesia, Intensive Care Medicine and Pain Medicine have to be acknowledged for their important contribution to this study. This study was supported by a grant of the Medical-Scientific Fund of the Mayor of 
Vienna (Bernhard Scheiner) and by a Gilead International Research Scholar Award (Thomas Reiberger).

The results were partly presented as a poster at the 8th International Symposium on Coagulation in Liver Disease, September 27-28, 2019, Groningen, The Netherlands.

Author contributions All authors were involved in the design of the study, acquisition of samples and/or analysis. PR, DMB and TR drafted the manuscript. All authors contributed to the critical discussion of the results and approved previous versions and the final version of the article, including the authorship list.

Funding Open access funding provided by Medical University of Vienna.

\section{Compliance with ethical standards}

Conflict of interest B.S. received travel support from AbbVie and Gilead. P.S. received speaking honoraria from Bristol-Myers Squibb and Boehringer-Ingelheim, consulting fees from PharmaIN, and travel support from Falk. M.M. served as a speaker and/or consultant and/ or advisory board member for AbbVie, Bristol-Myers Squibb, Gilead, and W. L. Gore \& Associates and received travel support from AbbVie, Bristol-Myers Squibb, and Gilead. T.R. received grant support from AbbVie, Boehringer-Ingelheim, Gilead, MSD, Philips Healthcare, Gore; speaking honoraria from AbbVie, Gilead, Gore, Intercept, Roche, MSD; consulting/advisory board fee from AbbVie, Bayer, Boehringer-Ingelheim, Gilead, Intercept, MSD, Siemens; and travel support from Boehringer-Ingelheim, Gilead and Roche. None of the remaining authors declares a conflict of interest for this manuscript.

Ethical approval All procedures performed in studies involving human participants were in accordance with the ethical standards of the institutional review board of the Medical University of Vienna (1262/2017) and with the 1964 Helsinki declaration and its later amendments.

Open Access This article is licensed under a Creative Commons Attribution 4.0 International License, which permits use, sharing, adaptation, distribution and reproduction in any medium or format, as long as you give appropriate credit to the original author(s) and the source, provide a link to the Creative Commons licence, and indicate if changes were made. The images or other third party material in this article are included in the article's Creative Commons licence, unless indicated otherwise in a credit line to the material. If material is not included in the article's Creative Commons licence and your intended use is not permitted by statutory regulation or exceeds the permitted use, you will need to obtain permission directly from the copyright holder. To view a copy of this licence, visit http://creativecommons.org/licenses/by/4.0/.

\section{References}

1. Tripodi A, Mannucci PM. The coagulopathy of chronic liver disease. N Engl J Med 2011;365(2):147-156

2. Lisman T, Porte RJ. Rebalanced hemostasis in patients with liver disease: evidence and clinical consequences. Blood 2010;116(6):878-885

3. Scheiner B, Northup PG, Gruber AB, Semmler G, Leitner G, Quehenberger P, et al. The impact of ABO blood type on the prevalence of portal vein thrombosis in patients with advanced chronic liver disease. Liver Int 2020;40(6):1415-1426

4. Mann KG. Thrombin formation. Chest 2003;124(3 Suppl):4S-10S
5. Dumitrescu G, Januszkiewicz A, Agren A, Magnusson M, Wahlin S, Wernerman J. Thromboelastometry: relation to the severity of liver cirrhosis in patients considered for liver transplantation. Medicine (Baltimore) 2017;96(23):e7101

6. Hugenholtz GCG, Lisman T, Stravitz RT. Thromboelastography does not predict outcome in different etiologies of cirrhosis. Res Pract Thromb Haemost 2017;1(2):275-285

7. Kleinegris MC, Bos MH, Roest M, Henskens Y, Ten Cate-Hoek A, Van Deursen C, et al. Cirrhosis patients have a coagulopathy that is associated with decreased clot formation capacity. $\mathbf{J}$ Thromb Haemost 2014;12(10):1647-1657

8. Lentschener C, Flaujac C, Ibrahim F, Gouin-Thibault I, Bazin M, Sogni P, et al. Assessment of haemostasis in patients with cirrhosis: relevance of the ROTEM tests? A prospective, cross-sectional study. Eur J Anaesthesiol 2016;33(2):126-133

9. Stegewerth K, Weber CF, Moehlmann M, Adam EH, Zacharowski $\mathrm{K}$, Zeuzem S, et al. Fast interpretation of thromboelastometry and aggregometry in patients suffering from chronic liver disease. Clin Lab 2019;65(8)

10. Tripodi A, Primignani M, Chantarangkul V, Viscardi Y, Dell'Era A, Fabris FM, et al. The coagulopathy of cirrhosis assessed by thromboelastometry and its correlation with conventional coagulation parameters. Thromb Res 2009;124(1):132-136

11. de Franchis R, VIF B. Expanding consensus in portal hypertension: report of the Baveno VI Consensus Workshop: Stratifying risk and individualizing care for portal hypertension. J Hepatol 2015;63(3):743-752

12. Ripoll C, Groszmann R, Garcia-Tsao G, Grace N, Burroughs A, Planas R, et al. Hepatic venous pressure gradient predicts clinical decompensation in patients with compensated cirrhosis. Gastroenterology 2007;133(2):481-488

13. Mandorfer M, Kozbial K, Schwabl P, Chromy D, Semmler G, Stattermayer AF, et al. Changes in hepatic venous pressure gradient predict hepatic decompensation in patients who achieved sustained virologic response to interferon-free therapy. Hepatology 2019

14. D'Amico G, Pasta L, Morabito A, D'Amico M, Caltagirone M, Malizia G, et al. Competing risks and prognostic stages of cirrhosis: a 25 -year inception cohort study of 494 patients. Aliment Pharmacol Ther 2014;39(10):1180-1193

15. Reiberger T, Puspok A, Schoder M, Baumann-Durchschein F, Bucsics T, Datz C, et al. Austrian consensus guidelines on the management and treatment of portal hypertension (Billroth III). Wien Klin Wochenschr 2017;129(Suppl 3):135-158

16. Moitinho E, Escorsell A, Bandi JC, Salmeron JM, Garcia-Pagan JC, Rodes J, et al. Prognostic value of early measurements of portal pressure in acute variceal bleeding. Gastroenterology 1999;117(3):626-631

17. Reiberger T, Ferlitsch A, Payer BA, Mandorfer M, Heinisch BB, Hayden $\mathrm{H}$, et al. Non-selective betablocker therapy decreases intestinal permeability and serum levels of LBP and IL-6 in patients with cirrhosis. J Hepatol 2013;58(5):911-921

18. Simbrunner B, Mandorfer M, Trauner M, Reiberger T. Gut-liver axis signaling in portal hypertension. World $\mathbf{J}$ Gastroenterol 2019;25(39):5897-5917

19. Wosiewicz P, Zorniak M, Hartleb M, Baranski K, Hartleb M, Onyszczuk M, et al. Portal vein thrombosis in cirrhosis is not associated with intestinal barrier disruption or increased platelet aggregability. Clin Res Hepatol Gastroenterol 2016;40(6):722-729

20. Payance A, Bissonnette J, Roux O, Elkrief L, Gault N, Francoz $\mathrm{C}$, et al. Lack of clinical or haemodynamic rebound after abrupt interruption of beta-blockers in patients with cirrhosis. Aliment Pharmacol Ther 2016;43(9):966-973

21. Reiberger T, Schwabl P, Trauner M, Peck-Radosavljevic M, Mandorfer M. Measurement of the Hepatic Venous Pressure Gradient and Transjugular Liver Biopsy. J Vis Exp 2020(160) 
22. D'Amico G, Morabito A, D’Amico M, Pasta L, Malizia G, Rebora $\mathrm{P}$, et al. Clinical states of cirrhosis and competing risks. J Hepatol 2018;68(3):563-576

23. Turco L, de Raucourt E, Valla D, Villa E. Anticoagulation in the cirrhotic patient. JHEP Rep 2019;1(3):227-239

24. Jindal A, Bhardwaj A, Kumar G, Sarin SK. Clinical decompensation and outcomes in patients with compensated cirrhosis and a hepatic venous pressure gradient $>/=20 \mathrm{~mm} \mathrm{Hg}$. Am J Gastroenterol 2020

25. Mandorfer M, Hernandez-Gea V, Garcia-Pagan JC, Reiberger T. Noninvasive diagnostics for portal hypertension: a comprehensive review. Semin Liver Dis 2020

26. Chau TN, Chan YW, Patch D, Tokunaga S, Greenslade L, Burroughs AK. Thrombelastographic changes and early rebleeding in cirrhotic patients with variceal bleeding. Gut 1998;43(2):267-271

27. Debernardi Venon W, Ponzo P, Sacco M, Mengozzi G, Raso S, Valpreda A, et al. Usefulness of thromboelastometry in predicting the risk of bleeding in cirrhotics who undergo invasive procedures. Eur J Gastroenterol Hepatol 2015;27(11):1313-1319
28. Mandorfer M, Schwabl P, Paternostro R, Pomej K, Bauer D, Thaler J, et al. Von Willebrand factor indicates bacterial translocation, inflammation, and procoagulant imbalance and predicts complications independently of portal hypertension severity. Aliment Pharmacol Ther 2018;47(7):980-988

29. Bos S, van den Boom B, Kamphuisen PW, Adelmeijer J, Blokzijl $\mathrm{H}$, Schreuder T, et al. Haemostatic profiles are similar across all aetiologies of cirrhosis. Thromb Haemost 2019;119(2):246-253

30. Bianchini M, De Pietri L, Villa E. Coagulopathy in liver diseases: complication or therapy? Dig Dis 2014;32(5):609-614

Publisher's Note Springer Nature remains neutral with regard to jurisdictional claims in published maps and institutional affiliations. 